\section{ENFOQUES POSITIVOS Y NORMATIVOS EN EL ESTUDIO DE LAS POLÍTICAS DE CIENCIA Y TECNOLOGÍA}

\author{
Pilar Rico-Castro \\ Fundación Española para la Ciencia y la Tecnología (FECYT) \\ pilar.rico@fecyt.es \\ Rafael Morera Cuesta \\ Consejo Superior de Investigaciones Científicas (CSIC) \\ r.morera@orgc.csic.es
}

\section{POSITIVE AND NORMATIVE APPROACHES IN SCIENCE AND TECHNOLOGY POLICY STUDIES.}

\begin{abstract}
The aim of this paper is to show how normative approaches to scientific research and technological development ( $R \& D)$ policies have shaped positive analysis. It presents a general overview of those contributions from decision makers and researchers to the early debates that founded social studies of research and development in mid 40s. Moreover, this article uses the distinction between positive and normative approaches to hold that international organizations, especially OECD, held the leadership in the normative construction of $R \& D$ discourses, and that subsequent positive analyses have been conducted in academia following normative guidelines. This applies for both national and international academic circles.
\end{abstract}

KEY WORDS: Scientific research and technological development policies, positive and normative approaches, OECD.

\section{INTRODUCCIÓN}

El objeto de este trabajo son los estudios sociales sobre las políticas de investigación científica y desarrollo tecnológico (I+D). El objetivo es el de presentar, de forma ordenada y coherente, aquellos trabajos que se han hecho para explicar cómo surgió esta área de acción pública en el contexto internacional y de qué manera, al nacimiento de un nuevo espacio de decisión gubernamental y de sus estándares de buenas prácticas, le siguió la apertura de un vasto campo de análisis para las ciencias sociales. El artículo distingue entre las aproximaciones normativas y positivas (Cordes, 1997), desde mediados del siglo XX hasta los primeros años del siglo XXI, y trata de poner de manifiesto cuáles han sido las relaciones de influencia mutua entre ambas
RESUMEN : Este artículo presenta una visión general de la evolución de los trabajos que estudian las políticas públicas de investigación científica y desarrollo tecnológico $(I+D)$, desde la apertura de este campo a la acción del gobierno en la década de 1940 hasta la actualidad. Tras hacer un repaso descriptivo de cuáles fueron las primeras aportaciones de los decisores públicos y de los académicos a este campo de conocimiento durante su proceso de creación, este trabajo utiliza la distinción entre enfoques positivos y normativos y defiende que el liderazgo normativo ha correspondido a los organismos internacionales, especialmente a la $\mathrm{OCDE}$, y que los enfoques positivos en la literatura académica internacional y en la nacional han evolucionado siguiendo las pautas marcadas por esta institución.

PALABRAS CLAVE: Políticas públicas de investigación científica y desarrollo tecnológico, enfoques positivos y normativos, OCDE.

corrientes. Con ello se pretende aportar una perspectiva diferente, una herramienta que ayude a una comprensión ordenada de toda la literatura que se ha escrito sobre este espacio de la acción pública y, por lo tanto, acercar a los estudiosos de este campo de conocimiento una forma de entender la evolución de las políticas a través de cómo han sido y de cómo se ha establecido que debian ser.

Los estudios académicos que se han desarrollado para tratar de explicar los distintos procesos que surgen alrededor de la actividad científica han sido multidisciplinares. La producción de nuevo conocimiento y su posterior aplicación práctica es una actividad que, si bien ha ocupado a los seres humanos a lo largo de la Historia, alcanzó una dimensión política, social y económica de relevancia ineludible a principios del siglo XX. Desde entonces y en la 
mayoría de las áreas que componen las ciencias sociales, la filosofía, la sociología, la historia, la economía y la ciencia política, se han ido abriendo espacios de comprensión y análisis centrados en los muy diversos objetos de estudio que han surgido con la institucionalización de la ciencia como actividad de progreso social y económico y la legitimación de la intervención del Estado en su regulación. Los desarrollos que se han hecho dentro de las distintas ramas, a pesar de las diferencias metodológicas en su aproximación al objeto de estudio, han resultado en los últimos años en cierta colisión disciplinaria.

La filosofía y la sociología de la ciencia han sumado esfuerzos en estudiar a los actores productores de ciencia y tecnología en su unidad más básica, que son los investigadores individuales y en colectivo. En este sentido, ambas disciplinas se han encontrado en el análisis de los mecanismos y procedimientos a través de los cuales los científicos elaboran su trabajo y establecen sus evidencias, de cuáles son sus procesos de observación, experimentación, recogida de datos empíricos, teorización sobre los resultados obtenidos, predicción de acontecimientos futuros basándose en la teoría y corroboración de los resultados, de cuáles son los mecanismos de validación o falsación de hipótesis, los acuerdos inter pares adoptados ante la necesidad de hacer crecer la disciplina, de las relaciones de los científicos en comunidad, así como de las relaciones entre éstos y la sociedad. Ámbitos tan decisivos como el establecimiento de jerarquías intelectuales y códigos de comportamiento derivados de éstas, la creación de espacios profesionales propios para cuyo ingreso se requiere un alto nivel de conocimiento técnico, el respeto a unas normas internas estrictas e iguales en todos los países, los procesos de reparto de prestigio entre los científicos, los itinerarios de formación y aprendizaje que culminan en un ineludible ritual de acceso a la comunidad o los procesos de comunicación de los resultados de la investigación a la sociedad, con los consiguientes debates éticos que esto pueda conllevar, constituyen los ámbitos de especialización en los que ambas disciplinas se han desenvuelto y han extendido sus ramificaciones mutuas.

Los economistas, en un primer momento dedicados a demostrar que la implicación de los países en actividades de investigación científica y desarrollo tecnológico es un factor de producción equiparable en pie de igualdad con el trabajo y el capital, así como a sostener la certeza empírica de que un mayor nivel de desarrollo científico genera riqueza y bienestar a medio y largo plazo y que las economías basadas en el conocimiento son hoy en día las más sostenibles y productivas del panorama internacional, cada vez toman más en cuenta los factores políticos y sociales tratados por otras disciplinas en sus aproximaciones al objeto de estudio. Así, se han unido a los sociólogos y a los politólogos en el análisis de cuáles son los mecanismos de capitalización de la actividad científica e innovadora, qué correas de transmisión utilizan los agentes de producción del conocimiento para maximizar el impacto positivo de su trabajo sobre la economía y la sociedad, así como en el estudio del diseño, la configuración y el cambio organizativo de los centros de investigación científica y tecnológica. Los mecanismos de conexión de las necesidades de las sociedades y las industrias en determinados campos científicos y tecnológicos con el trabajo de los laboratorios y centros de investigación públicos y privados ha sido también objeto de estudio para los economistas.

A pesar de esta riqueza de interrelaciones entre las distintas áreas de conocimiento de las ciencias sociales, se puede afirmar que los estudios realizados desde la ciencia política se distinguen por un objeto preferente de investigación y por prestar una mayor atención a algunos problemas sociales en este ámbito. Los politólogos han dedicado sus esfuerzos a estudiar las estructuras y las relaciones de poder que se dan alrededor de la actividad de producción de conocimiento. Su principal campo de trabajo ha sido el de las políticas públicas de ciencia y tecnología: quiénes son los actores involucrados en regular los mecanismos de fomento y coordinación de las actividades de I+D en todos los niveles en los que se diseñan políticas (nacional, regional e internacional), qué arquitectura institucional sostiene los procesos de regulación, fomento y decisión de los actores políticos, qué mecanismos de financiación pública se establecen, qué procesos Ilevan a la adjudicación de partidas presupuestarias a actividades de I+D y cómo estos fondos llegan finalmente a los investigadores, qué itinerarios y qué carreras profesionales se diseñan para el personal dedicado a la ciencia y la tecnología, el papel de los distintos grupos de presión, el establecimiento de prioridades, los procesos de toma de decisiones, así como los sistemas de incentivos diseñados para que las políticas funcionen son algunos de los campos de investigación académica más relevantes en esta área. 
Este trabajo se centra en estudiar aquellos que se han realizado sobre el análisis de la acción pública desde la ciencia política, distinguiendo entre los enfoques normativos, concepto que se refiere a las aproximaciones hechas desde el deber ser, y los enfoques positivos, que analizan la realidad tal y como se ha desenvuelto, sin atenerse a las categorias que las regulan o las canalizan. Para ello, este artículo se divide en cuatro partes bien diferenciadas. En primer lugar, se presenta una necesariamente breve panorámica de cómo surgieron las políticas de ciencia y tecnología como objeto de estudio y cuáles fueron los primeros debates de los que se ocuparon los trabajos que hoy conocemos como clásicos. En segundo lugar, se plantea el papel que desarrollaron los organismos internacionales, con especial interés sobre el rol de la Organización para la Cooperación y el Desarrollo Económico (OCDE), y su importancia en el establecimiento de cánones normativos de buen gobierno de la ciencia y la tecnología entre los países occidentales. En tercer lugar, se lleva a cabo un repaso cronológico de las principales aportaciones al análisis de las políticas de ciencia y tecnología realizadas desde el ámbito académico, tanto en el entorno internacional como en el nacional, y se establecen los puntos de conexión que explican su evolución como efecto de la influencia de las aproximaciones normativas. Por último, se presentan las conclusiones.

\section{Las políticas de Ciencia y tecnología COMO OBJETO DE ESTUdIO}

Las políticas públicas de ciencia y tecnología son todas aquellas decisiones y acciones intencionales adoptadas por los gobernantes con el objetivo de apoyar, promover o influenciar la dirección y el ritmo de desarrollo de la ciencia y la tecnología (Shils, 1968). Es decir, el conjunto de intervenciones directas de los poderes públicos, a través de programas específicos, mediante las cuales se influye sobre los elementos del sistema de producción de I+D y se modifican las condiciones bajo las que llevan a cabo su actividad los actores productores de investigación y desarrollo. Esto se materializa en decisiones que afectan, en primer lugar, a la gobernanza de la ciencia y la tecnología, dicho de otro modo, la arquitectura institucional y el marco legal en el que se inscriben la gestión y la regulación de estas actividades. En segundo lugar, a la adjudicación de mayores o menores recursos económicos y humanos a las actividades de investigación científica y desarrollo tecnológico frente a otro tipo de actividades de servicio público. En tercer lugar, al modo en que estos recursos se distribuyen entre las distintas disciplinas del saber, los tipos de programas y mecanismos especificos que se articulan para el reparto de fondos y los itinerarios profesionales que se establecen para los investigadores. En cuarto lugar, la tipología de organizaciones en las que los científicos desarrollan su labor investigadora y las dinámicas que se producen entre los grupos de investigación dentro de estos centros, que afectan a la socialización en pautas comunes de comportamiento, al aprendizaje del personal en formación, a las condiciones laborales a las que se enfrentan los científicos y tecnólogos a lo largo de su carrera profesional, así como las formas en las que los colectivos de investigadores gobiernan la república de la ciencia (Polanyi, 1962; Rip, 1994) y otorgan premios y recompensas a través del prestigio. Por último, las políticas públicas de ciencia y tecnología también inciden sobre cómo obtener retornos de las inversiones en actividades de $I+D$ y cómo rentabilizar social y económicamente este esfuerzo.

Algunos autores han fijado el nacimiento de la noción de política científica en el siglo XVII, época en la que ya había investigadores que trabajaban de forma individual al servicio de sus gobernantes en el desarrollo de materias especializadas como la cartografía, los sistemas de medición o el avance de la química (Ziman, 1984) y ya existian autores como Bacon o Condorcet que hablaban de la relación entre los científicos y los estados (Ronayne, 1984). Sin embargo, la ampliación de la expresión política científica y su definitiva transformación en política científica y tecnológica es muy posterior, ya que se sitúa a finales de la década de 1970 y principios de 1980 (Gummet, 1992). Este trabajo parte de la asunción de que las políticas públicas de ciencia y tecnología quedaron consolidadas como tales y se extendieron por Europa y Estados Unidos tras la Segunda Guerra Mundial, cuando se dio una confluencia de los intereses de los Estados, que tuvieron la oportunidad de comprobar el alcance que la capacidad científico tecnológica había tenido en la configuración geoestratégica y en el nuevo mapa de fuerzas internacional surgido tras la contienda bélica, y de los propios científicos, que comenzaron a reclamar a los gobiernos que se hiciesen cargo del apoyo institucional y financiero de sus actividades y su equipamiento, así como de la creación de estructuras organizativas sólidas

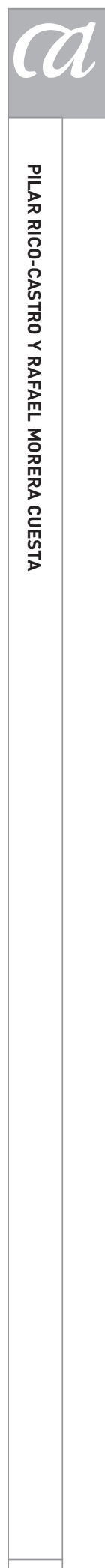

ARBOR CLXXXV 738 julio-agosto [2009] 793-807 ISSN: 0210-1963 
que dieran cobertura a su actividad investigadora (Price, 1954; Salomon, 1970; Gummet, 1992). Por lo tanto, la institucionalización de la ciencia y la tecnología como esferas de regulación legítima por parte de los poderes públicos a nivel internacional y el sometimiento, en mayor o menor grado, de las agendas de investigación de los científicos a las prioridades marcadas por las políticas son fenómenos que pertenecen de forma integra al siglo XX.

Asociados a esta nueva esfera de decisión pública, pronto se crearon instituciones encargadas de diseñar el buen hacer de la acción pública, que trabajaban bajo el consenso de que la ideología era una variable menor en el diseño de estas políticas, en las que debian primar las cuestiones técnicas sobre las doctrinarias (Brickman, 1981). Los primeros discursos normativos fueron elaborados por Estados Unidos, que pronto pasaron el testigo a organizaciones internacionales tales como la Organización de las Naciones Unidas para la Educación, la Ciencia y la Cultura (UNESCO), la Unión Europea (Comunidad Económica Europea CEE, desde 1957 hasta 1992) y la OCDE, que entraron a debatir sobre cómo habian de intervenir los gobiernos en el diseño de las políticas de ciencia y tecnología, cómo definir los conceptos relacionados con las actividades de investigación científica y desarrollo tecnológico y qué indicadores establecer para medir estas actividades.

Junto a ellas surgieron grupos de académicos que centraron su objeto de estudio en estas políticas y abrieron un vasto nicho de investigación dentro del cual comenzaron a generar una literatura especifica. Los primeros debates positivos que trataban de explicar la variedad y el alcance de las diversas formas de la acción pública fueron conducidos por académicos de diversas áreas de conocimiento, que recalaron en esta materia sin haber sido formados en una disciplina común ni contar con una metodología estandarizada de aproximación a su objeto de estudio. Debido a que era un cuerpo doctrinario en proceso de formación y al hecho de que los estudios sobre políticas de ciencia y tecnología se centran en particularidades propias de la naturaleza de la labor científica, se trataba de un campo de análisis que en sus inicios no estuvo reservado a los politólogos. Investigadores de muy diversa formación, entre los que se incluian científicos experimentales ${ }^{1}$ e incluso ingenieros con un muy variado bagaje intelectual, desarrollaron esta área desde sus respectivos campos (Brickman, 1981). A pesar de que este cuerpo de literatura tardaría algunos años en constituir su propio campo de conocimiento y de su falta de ortodoxia metodológica inicial, los análisis de políticas de ciencia y tecnología pronto se constituyeron en una parte esencial de los estudios sociales sobre ciencia y tecnología, y sus trabajos suscitaron un gran interés en los decisores públicos.

Este trabajo se centra en poner de manifiesto la especial incidencia en los países desarrollados que ha tenido la OCDE desde su creación en 1961, cuyas indicaciones han confluido en numerosas ocasiones con el ascenso de las políticas europeas a partir del gran desarrollo experimentado desde la década de 1990. Las directrices de esta organización internacional no sólo han apuntado las vías por las que debían desarrollarse las líneas políticas, sino que también han acuñado todo un marco conceptual cuya influencia ha sido decisiva en la producción de los modelos analíticos empleados por los académicos. Esto es, mientras que los foros normativos en el seno de la OCDE han evolucionado en la conceptualización de los modelos y de los entornos de producción del conocimiento, así como en las propuestas de acción pública, los académicos han utilizado esas estructuras conceptuales para articular su pensamiento y tratar de dar respuesta a la pregunta de cómo y por qué cambian las relaciones entre los científicos y los gobiernos.

\subsection{Los trabajos clásicos: primeros debates}

Desde el surgimiento de los estudios sobre las políticas de ciencia y tecnología hasta nuestros dias han transcurrido seis décadas. Los primeros trabajos académicos que tomaron la acción pública en esta materia como objeto de estudio se centraron en tratar de demostrar que, efectivamente, existía una relación entre el capital científico y tecnológico de los paises y su bienestar social para, posteriormente, argumentar a favor de que los gobiernos asumiesen la responsabilidad de financiar, y en cierto grado dirigir, la actividad de los científicos (Bernal, 1939; Mansfield, 1966; Tisdell, 1981; Ronayne, 1984).

Una de las primeras aportaciones en esta dirección fue la obra The Social Function of Science, publicada en 1939 por el científico irlandés de ideologia marxista John Bernal, en la que analizaba el sistema británico de ciencia para poner de manifiesto sus carencias financieras, organizativas, de coordinación y de sensibilidad hacia las necesidades de la sociedad. Bernal hacía un alegato ideológico a favor de la 
idea de que los poderes públicos debían involucrarse en las actividades de los científicos y abrió un nuevo frente de debate al defender que estas políticas no sólo debían existir sino que debían dirigir el trabajo de los investigadores a objetivos sociales específicos. Apenas esta obra vio la luz, Bernal pudo ver cómo su doctrina era llevada a la práctica, ya que el inicio de la Segunda Guerra Mundial tuvo como una de sus consecuencias que la política científica recibiese un fuerte impulso en países como Estados Unidos, el Reino Unido o Alemania, cuyo papel en la contienda bélica les planteaba la necesidad perentoria de desarrollar su capacidad armamentística a través de lo que se dio en llamar ciencia para la guerra (Ronayne, 1984).

Las enseñanzas obtenidas tras el desenlace de los acontecimientos de 1945 crearon un clima en el que ya no cabian dudas sobre la necesidad de que los poderes públicos entrasen a tutelar y financiar la actividad de producción científica, aunque las nuevas aportaciones pedian grados de libertad para los científicos muy superiores a los propuestos por Bernal. El informe Science, the endless frontier, escrito en 1945 por el senador norteamericano Vannevar Bush, y el impacto que causó sobre las políticas públicas estadounidenses ha sido considerado como el elemento que explica la legitimación del nuevo discurso que definía las relaciones entre el Estado y los científicos tras el conflicto bélico (Ronayne, 1984; Kleinman, 1995). El informe fue elaborado para ayudar a resolver la disyuntiva en la que se encontraba el gobierno norteamericano sobre qué rumbo debía tomar su política científica y tecnológica una vez terminada la guerra. Bush apuntaba de forma clara hacia la necesidad de mantener la investigación dirigida hacia fines militares en tiempos de paz, aunque reclamaba la importancia de fomentar la investigación básica civil, a pesar de su alto coste y sus resultados inciertos, a través de programas de financiación estables y a largo plazo. A diferencia de Bernal, defendía la necesidad de que los científicos gozasen de una autonomía total en la dirección de sus investigaciones y en el reparto de los recursos públicos para investigación fundamental, para lo cual abogaba por la creación de la National Science Foundation como organismo formado por científicos desinteresados que elaborasen e implementasen la política de ciencia y tecnología con fondos públicos (Bush, 1945).

En esta misma dirección apuntaba el trabajo The Republic of Science escrito en 1962 por el economista liberal británico de origen húngaro Michael Polanyi, en el que se hacía un alegato ideológico en contra de cualquier tipo de intromisión por parte del poder estatal en la creatividad de los investigadores. En su artículo, publicado en la revista Minerva y profusamente citado por la literatura especializada, Polanyi se posicionaba en contra de cualquier tipo de planificación y abogaba por que los científicos desarrollasen su labor de forma autónoma, guiados por su propia creatividad y no por las prioridades marcadas externamente por los gobiernos, en la creencia de que en ese marco de absoluta libertad se producirian los movimientos de coordinación espontánea entre iniciativas independientes que debían guiar la actividad investigadora (Polanyi, 1962).

Estos trabajos fueron desarrollados en el entorno académico y recogieron las bases argumentales en las que se sustentaron los grandes debates normativos sobre qué papel debía jugar la acción pública dentro de las políticas y sobre qué límites se debian trazar a la intromisión de los poderes públicos en la actividad investigadora. El resultado fue que se extendió la concepción de que la ciencia era el motor del progreso, de modo que, a pesar de que existían incentivos al avance de las cuestiones militares y científicas para el desarrollo de la gran ciencia, los decisores públicos pusieron en marcha modelos de producción de conocimiento basados en la oferta de los propios científicos, asumiendo el esquema lineal de investigación básica, según el cual la acumulación de este conocimiento desembocaría en el deseado cambio científico-tecnológico. Entre los científicos y el estado se estableció un contrato social de patronazgo y financiación de la autonomía científica, según el cual no era necesario articular una intervención pública directa sobre la actividad investigadora más allá de la financiación estable a través de recursos materiales y humanos, cuya distribución debía estar en manos de los propios investigadores. Esto desembocó en que se estableciesen mecanismos de toma de decisiones de abajo-arriba, controlados por los grupos de presión formados por científicos (Sanz-Menéndez y Santesmases, 1996; Moso, 1999).

Posteriormente, durante la década de 1990, se produjeron aportaciones muy importantes al estudio de los procesos que habian resultado en la cristalización de los sistemas de gobierno de la ciencia y la tecnología. Algunos autores emplearon el enfoque principal-agente para explicar la re- 
lación que se había forjado entre los estados (el principal) y los científicos (el agente) y sus aportaciones fueron de una gran riqueza analítica (Guston, 1996; Braun, 1998; Van der Meulen, 1998). Guston (1996) fue el primer autor que introdujo este enfoque analítico, sobre el que se basó para defender que el debate clave al que tenían que enfrentarse los decisores públicos a la hora de construir los incipientes sistemas públicos de ciencia y tecnología era el grado de delegación de autoridad que los gobiernos debían ceder a los científicos. El trabajo de Van der Meulen (1998) recogió el testigo y se centró en explicar que, si bien existe una relación principal-agente entre todos los estados y sus colectivos de investigadores, esa relación puede tener un perfil muy diferente y cristalizar en instituciones distintas. Para este autor, el punto en el que los decisores públicos y los investigadores encuentran la estabilidad en su relación de intercambio de fondos por conocimiento dependerá del juego político, de las asunciones, de las preferencias y del grado de interdependencia de los actores entre sí. El nivel de estabilidad en esta relación se ve reflejado en las instituciones que gobiernan la ciencia y en las organizaciones intermediarias surgidas entre ambos grupos, que, una vez creadas, son difíciles de cambiar debido al peso de la inercia y de las rutinas de funcionamiento que operan con especial fuerza en las relaciones entre los gobiernos y los científicos.

Por su parte, Braun (1998) se centró en estudiar la importancia específica de un tipo de organización intermediaria a través de la que se canalizan las relaciones entre los científicos y los estados: los consejos de investigación entendidos como agencias financiadoras que adjudican los fondos públicos a las actividades de investigación científica. Este trabajo asumía la idea lanzada por Rip (1994) sobre que las agencias de financiación de la actividad investigadora son parte esencial de la república de la ciencia y las situó a medio camino entre los decisores y los ejecutores de la $I+D$, como las organizaciones intermediarias clave de los sistemas de ciencia y tecnología. La principal aportación de Braun en este trabajo fue poner de manifiesto que las instituciones financiadoras juegan un papel crucial en la dirección en la que avanzan las distintas disciplinas, ya que, al canalizar las preferencias de los decisores gubernamentales y manejar la financiación pública, logran influir no sólo sobre las estrategias colectivas de los investigadores sino también sobre el propio desarrollo cognitivo del conocimiento científico.

\subsection{La importancia de las organizaciones internacionales y el liderazgo normativo de la OCDE}

La discusión inicial sobre el papel que la política debía jugar en relación con la ciencia continuó siendo citada de forma ineludible en la literatura especializada y volvió a reabrirse sin apenas solución de continuidad por los discursos normativos de los gestores y decisores públicos. Paralelamente al desarrollo de los primeros debates, el gobierno de EEUU inauguró la creación de agencias de financiación para la investigación científica y el desarrollo tecnológico, poniendo así los primeros cimientos de la construcción de los discursos normativos en materia de gestión pública de la ciencia y la tecnología. El National Health Institute (NHI), convertida en la agencia federal para la investigación médica, la National Science Foundation (NSF), creada en el año 1950 con el objetivo de que fuese el órgano encargado de diseñar la política científica y de apoyar, promover y evaluar la investigación básica de carácter civil norteamericana, y la National Aeronautic and Space Administration (NASA) compusieron el elenco de agencias de financiación de la actividad investigadora en EE.UU. Con ellas cristalizó el primer diseño dominante en el establecimiento de mecanismos formales de relación entre el gobierno y los científicos, y esto otorgó a los EEUU una amplia ventaja respecto a Europa en el control de los modelos normativos a ambos lados al Atlántico (Ruivo, 1994; Kraemer, 2006)².

Sin embargo, a pesar de este protagonismo inicial de los Estados Unidos, canalizado fundamentalmente a través de la NSF, el diseño de las formas de organización de las relaciones entre los científicos y los gobiernos y el establecimiento de modelos normativos de las primeras políticas de ciencia pronto fue dominado por los organismos internacionales. El debate sobre cómo articular el papel de los políticos y sus decisiones con la actividad de los científicos quedó definitivamente instalado en el entorno internacional con la creación de la UNESCO en $1946^{3}$ y, sobre todo, con el nacimiento en 1961 de la OCDE. Originariamente, la UNESCO desarrolló labores de asistencia a los científicos y a sus organizaciones y posteriormente derivó su actividad a apoyar, dentro de los paises que lo necesitasen, la investigación orientada al desarrollo tecnológico (Fernández Carro, 2002; Drori et al., 2003), mientras que la OCDE fue ganando terreno hasta convertirse en la institución en la 
que los gobiernos de las principales economías occidentales se reunian periódicamente para establecer un marco de referencia y una doctrina común a la hora de elaborar sus políticas de ciencia y tecnología (Elzinga y Jamison, 1995) ${ }^{4}$. Actualmente, ambas organizaciones mantienen un importante rol como referencias en la recogida, homologación y distribución de indicadores sobre ciencia, tecnología e innovación a través de publicaciones periódicas como son el Science and Technology Statistical Yearbook de la UNESCO, o el Science, Technology and Industry Scoreboard, el Science, Technology and Industry Outlook y el Main Science and Technology Indicators, todos ellos de la OCDE.

En este trabajo se defiende la supremacía de la OCDE A través de sus informes y sus diagnósticos, este organismo internacional trabajó desde un principio por que se establecieran unas pautas comunes en la identificación de problemas que marcasen las agendas de decisión de los países miembros. Su capacidad de influencia quedó patente en el año 1964, cuando la OCDE celebró un seminario en la ciudad italiana de Frascati en el que se elaboró el Manual para la medición de las actividades de $1+D$, más conocido como el Manual de Frascati. Este documento se convirtió en el manual de referencia en el que quedaron establecidos los conceptos básicos y las clasificaciones canónicas de todos los elementos relacionados con la actividad de producción de conocimiento, así como la forma de medir cada uno de estos conceptos. Posteriormente fue completado con el Manual de Oslo para la recogida e interpretación de datos sobre innovación, publicado en el año 1992 junto con la Oficina Estadística Europea EUROSTAT y revisado y reeditado en 2005, el Manual de Patentes para el correcto uso de los datos sobre patentes como indicadores de $I+D$, publicado en 1994, y el Manual de Canberra para la medición de los recursos humanos destinados $a I+D$, publicado en 1995.

Dejando a un lado la producción de manuales por parte de la OCDE y su indudable peso en el establecimiento de definiciones estandarizadas y formas consensuadas de medir la ciencia y la innovación, el primero de los informes publicados en esta materia fue el trabajo Science and the Politics of Government, elaborado por un grupo de científicos y economistas para informar al Secretario General de la OCDE sobre qué cuestiones relacionadas con la ciencia y la tecnología requerían atención política. Este informe, que vio la luz en 1961 y que fue desde un principio conocido como el Informe Piganiol por haber sido coordinado por el francés Pierre Piganiol, ponía sobre la mesa la idea de que los recursos destinados a financiar la ciencia no eran ilimitados y que, por lo tanto, los estados debian diseñar políticas públicas que hiciesen posible una gestión eficaz y una asignación racional de los recursos (OECD, 1961). Se introdujo así la idea de que las actividades de producción de conocimiento debían ser objeto de medidas articuladas y específicas en pie de igualdad con otros ámbitos de la acción pública, lo que se dio en llamar políticas para la ciencia. Además, este informe trataba de conjugar la libertad de creación de los científicos, que se había impuesto en la etapa anterior, con la creciente necesidad de involucrar a los investigadores en actividades que diesen cobertura a las demandas técnicas de los estados, lo que se dio en llamar ciencia para la política (Sanz-Menéndez 1997, 84).

Una década después, en 1971, la OCDE sacó a la luz Science, Growth and Society, documento conocido como el Informe Brooks, en el que se daba un nuevo giro normativo a la acción pública en materia de investigación científica y desarrollo tecnológico. En este informe se ponía en duda la asunción de que toda actividad científica era per se positiva para la sociedad, debido a que entraron en escena nuevos problemas relacionados con la protección del medio ambiente y con la transparencia y la responsabilidad del colectivo de investigadores y tecnólogos (OECD, 1971). Este documento recogía las crecientes demandas de la sociedad por que la labor de los científicos adquiriese mayores niveles de responsabilidad social, tanto en lo que se refería al contenido de su actividad como a sus resultados, dando paso a lo que algunos autores han dado en llamar la repolitización de la ciencia (Elzinga y Jamison 1995, 113). Además, en el informe se recogian afirmaciones que apuntaban a que la relación entre inversión de recursos en investigación y desarrollo económico no era tan directa como se había creído hasta ese momento sino que encerraba grandes dosis de incertidumbre que los gobiernos debian tratar de controlar a la hora de diseñar sus políticas. Esta nueva aportación de la OCDE, además, aplicaba la distinción entre ciencia básica y ciencia aplicada definida en el Manual de Frascati, y argumentaba que esta última debía estar enfocada hacia objetivos sociales concretos que se alejasen de los aspectos relacionados con la defensa, poniendo un mayor énfasis en la ciencia para la política (Gummet, 1992; Sanz-Menéndez, 1997, 89; Godin, 2007, 15). 
Las mayores contribuciones normativas aportadas en la década de 1980 se recogieron en el informe Science and Technology Policy for the 1980s, publicado en el año 1981. En este documento, la OCDE instaba a las organizaciones de producción de conocimiento eminentemente básico a acercarse al mercado. Para ello, marcaba como prioridades la creación de vínculos entre la industria y las universidades, así como el enfoque de la acción pública hacia el desarrollo de nuevas tecnologías que diesen cobertura a las demandas de la política industrial. En este contexto, se promocionó la prospectiva como herramienta eficaz de diseño de las políticas de ciencia y tecnología. Se trataba de un sistema de establecimiento de prioridades novedoso, basado en la observación sistemática a largo plazo de la ciencia y la tecnología, enfocado a diseñar escenarios de futuro y a reducir la incertidumbre sobre la evolución de las distintas esferas del conocimiento y sus aplicaciones específicas, en el que participan miembros destacados de las comunidades de científicos y tecnólogos, y que permitía dar un giro comercial a la producción de conocimiento (Elzinga y Jamison, 1995, 117).

A mediados de la década de 1990, la OCDE puso en circulación la noción de Sistema Nacional de Innovación (Godin, 2007), con el que las concepciones tradicionales sobre los procesos de producción de conocimiento y su posterior transformación en progreso económico y social adquirieron una dimensión más adecuada a su complejidad. La idea de sistema nacional de innovación, acuñada por Freeman (1987), Lundvall (1992) y Nelson (1993), se deriva de la asunción de que la actividad investigadora tiene como último objetivo generar innovación y que los investigadores forman parte de un entramado superior en el que comparten su espacio con los órganos de decisión, de planificación y coordinación de las políticas de ciencia y tecnología, con los órganos de financiación de sus actividades, con otros actores productores de conocimiento del sector público, privado o universitario, con los órganos de evaluación y de apoyo a sus actividades y con el entorno social, político y económico en el que todos ellos se insertan. Esta constelación de actores y la riqueza de relaciones que se establecen entre ellos fue señalada por la OCDE en 1997 en su informe National Innovation Systems y en 1999 en el documento Managing National Innovation Systems. En ambos se apuntaba que las coordenadas para una política de éxito pasaban por fomentar el dinamismo del sistema, potenciando la participación del sector privado en la financiación y ejecución de las actividades de producción científica y tecnológica, y diseñando medidas de capitalización del conocimiento y de transferencia de tecnología a los sectores productivos. Junto a este nuevo giro normativo de las aportaciones de la OCDE surgió además la necesidad de diseñar nuevos indicadores que fuesen capaces de captar el rendimiento de los procesos de distribución de conocimiento entre los actores del sistema y que midiesen las nuevas exigencias de ejecución de la innovación (Godin, 2007).

\subsection{La evolución positiva de las políticas de ciencia y tecnología}

Una de las primeras formas de estudiar las políticas de ciencia y tecnología, que pronto se extendió entre la comunidad académica, fue la de hacer revisiones sobre su evolución positiva con el objetivo de establecer periodos en su desarrollo. A pesar de que algunos autores desarrollaron estos trabajos a escala nacional, como es el caso de Dickson (1984), que se centró en establecer las fases por las que había pasado la política científica en Estados Unidos, la mayoría de ellos trataron de entender la evolución de estas políticas en los países desarrollados como un proceso que se había dado de forma más o menos paralela a escala internacional. En este marco de comprensión internacional se inscribieron los trabajos de Salomon (1970), Gummett (1992), Solingen (1993), Ruivo (1994) y Elzinga y Jamison (1995). Éstos han sido considerados por la literatura especializada como los autores clásicos que aportaron los primeros análisis a los cambios en la relación entre los científicos y el Estado.

El trabajo de Ruivo (1994) defiende que la política científica ha sufrido un proceso de internacionalización que explica que en los distintos países del entorno occidental se hubiesen dado pautas comunes en el desarrollo de esa área de regulación pública, lo que ella llama paradigmas de la política científica. Para esta autora, este proceso es consecuencia del importante papel que han tenido las organizaciones internacionales como OCDE, la UE (antes CE) o las agencias de Naciones Unidas en diseminar el conocimiento empírico sobre nuevos asuntos y nuevos instrumentos de regulación, así como creencias comunes sobre qué políticas debían implementarse para alcanzar los objetivos de progreso fijados por cada país. 
El trabajo de Elzinga y Jamison (1995) se ha constituido en una de las referencias ineludibles para los autores que posteriormente centraron su objeto de estudio en el análisis especifico de políticas concretas. En él se hace frente a las preguntas de investigación relativas a cómo y por qué han cambiado las agendas de política científica de los distintos paises durante la segunda mitad del siglo XX, y encuentra respuesta en el perfil de las élites encargadas de la gestión de las políticas, lo que dieron en denominar las culturas de las políticas. Para estos autores existe una cultura burocrática, dominada por las fuerzas de la administración del Estado y el ejército, una cultura académica, dominada por los científicos, una cultura económica, correspondiente a los sectores empresariales, y una cultura cívica, representante del poder de los movimientos sociales. Este trabajo defendía que el equilibrio de fuerzas entre estas cuatro culturas y la evolución de sus pesos relativos a lo largo de los años marcó las distintas etapas en la evolución de las políticas de ciencia y tecnología. Además, puso de manifiesto la existencia de una heterogeneidad en las pautas y en las preferencias de los distintos países del entorno occidental, lo que explicaron como una consecuencia de las dinámicas del entorno internacional.

Tras la publicación y difusión del Informe Piganiol, durante la década de 1960 se produjeron importantes aumentos presupuestarios en las partidas estatales destinadas a financiar las actividades de ciencia y tecnología. Este aumento de recursos fue destinado a financiar objetivos de investigación específicos y se vio además acompañado por un fortalecimiento de las medidas de control sobre la acción de los científicos. El Informe Brooks, por su parte, orientó la acción pública en materia de investigación científica y desarrollo tecnológico durante la década de 1970 hacia objetivos marcados por los movimientos sociales ecologistas, feministas y pacifistas que en aquellos años habían alcanzado una gran fuerza política, otorgando una supremacía a la cultura cívica sobre la burocrática y económica que habían tratado de dominar las esferas de decisión en la etapa anterior (Elzinga y Jamison, 1995). Autores como Brickman (1981) han señalado que durante estas dos décadas los análisis positivos sobre las políticas estuvieron excesivamente enfocadas hacia la financiación pública de la ciencia y la tecnología, en la creencia errónea de que las partidas presupuestarias destinadas a tal fin eran la única expresión de las preferencias gubernamentales.
Las revisiones de los trabajos (Moso, 1999) apuntan al hecho de que las políticas que se elaboraron durante esta época, y que recibieron el nombre de políticas de $I+D$, estaban articuladas sobre una concepción de la ciencia como mecanismo de solución de problemas. A diferencia de la etapa de los debates iniciales sobre el papel del Estado en la actividad investigadora, donde la producción de conocimiento respondía a la oferta de los propios científicos, en estos años la investigación estuvo movida por la fuerza de la demanda de los estados, que adoptaron el llamado modelo lineal tecnológico como modelo de cambio científico-tecnológico. En este contexto, el tipo de actividad desarrollado con más intensidad era la investigación aplicada, aunque con grandes matices y diferencias según los distintos contextos nacionales. También se invirtieron los términos en los mecanismos de toma de decisiones respecto a la etapa anterior y se establecieron procesos de arriba-abajo dominados por los decisores públicos. Esto hizo posible que las prioridades de la acción pública se reorientasen hacia cuestiones económicas y sociales, utilizando modelos de financiación selectivos, distribuidos y controlados por instituciones públicas que se diseñaron para cumplir esta misión. Además, como consecuencia de la publicación del Manual de Frascati, en todos los países comenzaron a contabilizarse las actividades de investigación científica y desarrollo tecnológico, desarrollando indicadores para poder medir tanto los inputs como outputs de las políticas.

Durante la década de 1980 los valores sociales perdieron toda su fuerza dentro de las prioridades gubernamentales en materia de ciencia, dejando paso a nuevos rumbos marcados por los valores de la cultura económica (Elzinga y Jamison, 1995). Los decisores públicos orientaron las nuevas políticas hacia objetivos económicos e industriales concretos, y aceptaron la planificación y la prospectiva como métodos habituales de toma de decisiones en la mayoría de los países. El nuevo rumbo que marcó la etapa desde mediados de la década de 1980 hasta mediados de la década de 1990 se caracterizó por que las políticas estaban orientadas a potenciar la tecnología y la innovación. A nivel internacional, la ciencia pasó a considerarse como una fuente de oportunidad estratégica, y la acción pública en esta materia se articuló sobre el llamado modelo de innovación sistémico de cambio científico-tecnológico. La producción de conocimiento se realizaba tanto a través de investigación básica como de investigación aplicada, y la

ARBOR CLXXXV 738 julio-agosto [2009] 793-807 ISSN: 0210-1963

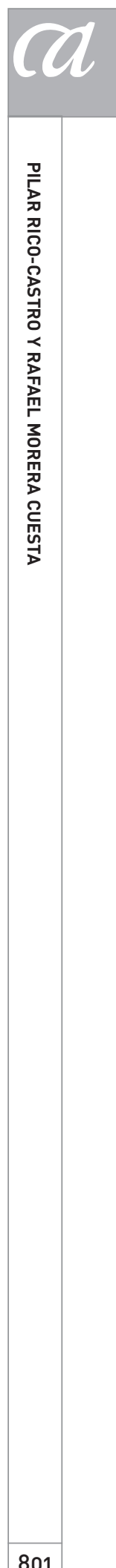


fuerza motriz de esta producción se basó tanto en la oferta de conocimiento emitida por los científicos como en la demanda de los estados. Esto repercutió en los mecanismos de toma de decisión que se institucionalizaron, en los que se combinaron procesos de abajo-arriba, liderados por los científicos, con procesos de arriba-abajo, liderados por los decisores públicos. El contrato social entre los científicos y el estado quedó redefinido en función de la capacidad de gestión de los recursos, de modo que la financiación pública se tornó muy selectiva, y los indicadores de inputs y outputs se desarrollaron y se ampliaron para poder evaluar de forma adecuada el rendimiento de las políticas (Moso, 1999).

Más allá de los elementos comunes, los distintos estados pusieron en marcha políticas específicas dentro de sus distintos contextos nacionales cuyo perfil se adaptó a las pautas normativas del contexto internacional. Sin embargo, a principio de la década de 1980 se alzaron algunas voces que ponían de manifiesto los escasos análisis sobre las políticas de ciencia y tecnología disponibles en la literatura académica (Brickman, 1981). El mayor volumen de información en este sentido proviene de la propia OCDE que, además de haberse hecho con el liderazgo de los discursos normativos, también dedicó importantes esfuerzos a realizar estudios de caso de las políticas puestas en marcha por los distintos paises, haciendo acopio de un importante capital de información a partir del cual podian identificar tendencias globales. Tan sólo en esta década la OCDE publicó trabajos descriptivos sobre las políticas de ciencia y tecnología en Grecia, Noruega, Portugal, Australia, Dinamarca y la antigua Yugoslavia.

En la década de 1990 cobraron fuerza los autores que reclamaban el peso del entorno nacional para explicar políticas que eran del dominio de los estados. En esta etapa, en la que los valores comerciales se habían extendido por la comunidad académica, los programas militares estaban siendo cuestionados y la cultura cívica estaba en desventaja respecto a las demás, los contextos nacionales ganaron fuerza a pesar de las referencias internacionales comunes (Elzinga y Jamison, 1995). Estos autores argumentaban que, puesto que las competencias de elección, en lo que se refiere a la asignación de recursos, de patronazgo, relativas al dirigismo ejercido sobre la labor de investigación de los científicos, y de control sobre los fines que deben alcanzarse mediante la investigación (Ziman, 1984) habian surgido y se habían desarrollado en la esfera de actuación de los gobiernos nacionales, la literatura estaba en deuda con la necesidad de construir explicaciones sobre los cambios en las políticas de ciencia y tecnología basadas en el ejercicio de estas tres funciones por parte de los gobiernos. En este contexto, surgieron autores en Europa y EEUU (Kleinman, 1995) que centraron sus esfuerzos en hacer análisis de las políticas públicas de I+D que se llevaban a cabo en los distintos contextos nacionales desde la perspectiva clásica del análisis de políticas públicas (Meny y Thoening, 1992).

Los trabajos que se realizaron con posterioridad se inscribieron en un nuevo paisaje, en el que la acción pública sobre la ciencia y la tecnología ha dejado de ser una competencia exclusiva de los gobiernos centrales. Poco a poco, junto al contexto nacional han surgido nuevas esferas de decisión. En Europa se ha dado un doble proceso de internacionalización y regionalización, resultado del liderazgo adquirido por las instituciones de gobierno de la Unión Europea en el fomento, promoción y desarrollo de la actividad científico-tecnológica de sus estados miembros, y de la creciente tendencia a la regionalización de este tipo de actuaciones públicas en los distintos países, independientemente de su carácter federal o unitario. Así, el análisis de las políticas públicas de I+D ha contado con nuevos y muy variados espacios de estudio, cuyo surgimiento ha coincidido con la implantación definitiva entre la literatura especializada del concepto de Sistemas Nacionales de Innovación (Lundvall, 1992; Nelson, 1993; Godin, 2007) y su posterior aplicación a la esfera regional a través del concepto de Sistemas Regionales de Innovación (Cooke et al., 1997).

Por otra parte, el proceso de creciente internacionalización de las actividades de investigación científica y desarrollo tecnológico también ha recibido atención particularizada dentro de la literatura académica (Guzzeti, 1995; Caracostas y Muldur, 1998). El estudio de Guzzeti goza de singular importancia porque hace un repaso detallado de la historia y la evolución de la política científica de la Unión Europea (entonces aún Comunidad Europea) desde 1948 hasta 1993. En su estudio, Guzzeti respeta un escrupuloso orden cronológico para describir las políticas de ciencia y tecnología adoptadas, en primera instancia, por las tres comunidades primigenias $y$, posteriormente, por las unión de las tres y el nacimiento de la Comunidad Económica Europea, tratando de poner el énfasis en sus procesos de 
toma de decisiones y en el peso de las diferentes instituciones comunitarias en el desarrollo de la acción pública a nivel europeo.

Posteriormente, en el nuevo espacio dominado por el concepto de sistemas nacionales de innovación, ha habido trabajos que han estudiado las diferencias entre los estados y sus políticas, considerando el espacio europeo como un sistema de innovación más. En este marco se inscribe el trabajo de Larèdo y Mustar (2001), que no sólo representa la contribución más importante en este sentido sino que supone una aportación a los estudios comparados sobre políticas de investigación científica y desarrollo tecnológico de gran valor añadido, debido a la falta de trabajos académicos de línea comparativa.

A través de las aportaciones hechas por los autores de los distintos países europeos y de EE.UU., estos autores señalan cómo a comienzos del siglo XXI los estados han comenzado a dedicar cada vez más esfuerzos a las labores de coordinación, y no tanto de regulación estricta, entre los diversos elementos de los sistemas nacionales de innovación. El surgimiento de las dimensiones regional e internacional en las políticas de ciencia y tecnología y la nueva concepción de los entornos de la I+D como sistemas complejos requiere por parte de los decisores nacionales de una importante labor de coordinación, que amortigüe las tensiones entre los procesos de globalización y regionalización de las políticas y articule la acción de los distintos sectores. Así, el sector público está siendo objeto de especial atención en los distintos países, donde la universidad se ha convertido en una prioridad común y los grandes centros públicos de investigación han sido objeto de reforma (Prest et al., 2003). En lo que se refiere al sector privado, las empresas de tamaño mediano y pequeño han ganado importancia como principales valedoras del sector empresarial, debido a que las grandes empresas han recorrido el camino a convertirse en multinacionales para poder responder al reto de la competitividad internacional y su papel dentro de los sistemas nacionales de innovación se ha complicado.

\subsubsection{Los estudios de las políticas de ciencia y tecnología en España}

En España, los trabajos dedicados a estudiar las políticas públicas que han configurado el sistema español de ciencia y tecnología se llevaron a cabo con un importante retraso respecto a los estudios realizados en el entorno internacional, como consecuencia del tardío desarrollo de este campo de actuación pública en nuestro país (González de la Fe et al., 2007).

Las primeras aproximaciones de autores nacionales a este objeto de estudio fueron de carácter descriptivo y surgieron en un contexto en el que se necesitaba conocer cuál era el estado del sistema de ciencia y tecnología para poder diseñar la que posteriormente sería la Ley 13/1986, de Fomento y Coordinación General de la Investigación Científica y Técnica, más conocida como la Ley de la Ciencia. Estos trabajos cubrieron espacios de conocimiento parciales que ayudaron a dibujar el panorama en el que se iban a poner los primeros cimientos del sistema, y sin duda llevaron a cabo una primera labor de acercamiento y sensibilización de la comunidad académica que hizo posible el posterior desarrollo de esta disciplina en nuestro país. Hubo aportaciones que trataron de poner de manifiesto el papel que juega la investigación científica sobre el desarrollo de los países (Mayor Zaragoza, 1982), otras que ahondaron sobre experiencias concretas de política científica en el marco del Consejo Superior de Investigaciones Científicas (Nieto, 1982), otras que centraron su atención en ver cómo se podrian implementar las formas de financiación y evaluación de la actividad científica (Sebastián, 1985) y otras que hicieron un diagnóstico de situación a partir del análisis de los indicadores básicos de la I+D en España (Muñoz y Ornia, 1986).

Una vez aprobada la Ley de la Ciencia y tras el lanzamiento del primer gran instrumento de la política científica de nuestro país, el Plan Nacional de Investigación y DesarroIlo, surgieron nuevas aportaciones que ponian de manifiesto un creciente interés por la materia, tanto en el ámbito académico como en el político (Dorado et al., 1991; López Piñero, 1991; Lafuente y Oro, 1992; Bellavista et al., 1998), así como otras que representaron las primeras evaluaciones de políticas y de programas concretos (Quintanilla, 1992; González de la Fe et al., 1996, 1997). El análisis de Luis Sanz-Menéndez sobre las políticas de ciencia y tecnología desarrolladas en nuestro país entre los años 1939 y 1997 supuso la aportación de mayor relevancia en esta línea, al realizar un exhaustivo estudio sobre las ideas, las instituciones y los intereses que habian guiado estas políticas durante todo este período. Este autor puso toda la carga explicativa en las dinámicas de lucha por el 
poder entre los distintos actores, asumiendo que las políticas de ciencia y tecnología, independientemente de lo específico de su contenido y de la bondad de sus objetivos, responden a decisiones de naturaleza política, y como tales han de entenderse. En este sentido, sus aportaciones se alejaban de aquellas que habian proclamado que, dadas las particularidades de la actividad de los científicos y la alta complejidad técnica de este tipo de decisiones, la acción pública en este ámbito estaría al margen de las luchas de poder de otras esferas de decisión y desprovista de dogmatismos doctrinarios.

Además de estos trabajos, el hecho de que en España el surgimiento de políticas regionales de ciencia y tecnología haya sido un proceso que se ha dado con especial fuerza y que las Comunidades Autónomas hayan recibido del Estado Central las competencias sobre el gobierno de las universidades y sobre el desarrollo y la promoción de la I+D dentro de sus territorios, ha propiciado que se desarrollase un vasto campo de análisis y estudios sobre las políticas regionales de ciencia y tecnología (Moso, 1999; Olazaran y Gómez Uranga, 2001; Sanz-Menéndez y Cruz-Castro, 2005; Rico-Castro, 2007). En este sentido, el trabajo Sistemas Regionales de Innovación editado por Mikel Olazaran y Mikel Gómez Uranga constituye una referencia ineludible porque recoge las aportaciones más importantes que se han hecho al análisis de las políticas regionales de I+D que se han realizado en España, aunando la concepción sistémica de los sistemas regionales de innovación con los análisis detallados de las políticas catalana, valenciana, navarra, andaluza, gallega, madrileña y vasca.

\section{Conclusiones}

Las políticas de ciencia y tecnología han variado considerablemente a lo largo de los años. Del mismo modo, los trabajos académicos que han tomado esta área de acción pública como su objeto de estudio también han evolucionado con el paso del tiempo. Los discursos positivos y normativos que han desarrollado los análisis de políticas de I+D han ido de la mano en su evolución a lo largo de las décadas del último medio siglo.

Los diseños de las políticas han tenido desde principios de la década de 1960 un referente normativo ineludible en la doctrina proveniente de la OCDE. Este organismo internacional contribuyó de forma decisiva al establecimiento de los fundamentos de las políticas de ciencia y tecnología, así como al diseño y desarrollo de sus instrumentos de medición en los países occidentales. Por ello, muchos de los primeros trabajos académicos que se hicieron con el objetivo de analizar estas políticas ponían el acento en el entorno internacional como factor explicativo de los cambios en las políticas nacionales. A esta situación contribuyó la falta de consenso metodológico y analítico en los primeros trabajos, que fueron realizados por académicos de muy diversas disciplinas, así como el hecho de que la propia OCDE también llevase a cabo una extensa labor de estudios de caso sobre las políticas de los distintos países.

Hasta la década de 1990 no se produjo la independencia de los enfoques positivos respecto a los normativos. En estos años surgieron autores formados en el análisis de las políticas públicas que analizaron las políticas de ciencia y tecnología dentro de su contexto nacional, siguiendo unas pautas metodológicas que se inscriben dentro de ortodoxia de la ciencia política. A partir de ahí comenzó a crearse un campo de conocimiento sólido en el que han ido floreciendo las aportaciones. A mediados de los años 1990 surgieron conceptos como el de sistema nacional de innovación, y se dieron procesos clave como fue el nacimiento de políticas regionales e internacionales de ciencia y tecnología que han ampliado sobremanera este campo de trabajo y han marcado definitivamente el devenir de los análisis de estas políticas.

Actualmente, la literatura especializada se encuentra ante el panorama más desafiante que se podría imaginar. Las líneas de investigación tienen un vastísimo campo abierto en los estudios comparados tanto en el ámbito regional como en el internacional, así como en la gran asignatura pendiente que son las evaluaciones del impacto de las políticas sobre los elementos del sistema. Si el área de conocimiento mantiene la línea de progreso en calidad de los trabajos y en crecimiento de los grupos de investigación que ha tenido hasta ahora, pronto asistiremos a un cambio de roles en lo que se refiere a los enfoques normativos y positivos, en el que los académicos se harán con el liderazgo del deber ser en materia de investigación científica y desarrollo tecnológico, garantizando así la supervivencia de este colectivo de intelectuales más allá de las fronteras del mundo académico. 


\section{NOTAS}

1 Éste es el caso de académicos como John Ziman, en el ámbito anglosajón, o de Emilio Muñoz, en el ámbito nacional.

2 Sobre la especial relevancia que tuvo la National Science Foundation en la construcción de las prácticas de intervención en $I+D$ en España, véase Fernández Carro, 2002.

3 Véase Mayor Zaragoza, 1996.

4 Sobre el contexto político de la creación de la National Science Foundation y la OCDE y su evolución histórica, véase Sanz-Menéndez, 1997.

\section{BIBLIOGRAFÍA}

Bellavista, Joan; Turpin, Tim y De Miguel, Jesús (1998): "Cultura organizativa de investigadores y entorno político y social", Papers, Revista de Sociología, n. ${ }^{5}$ 54, pp. 79-109.

Bernal, John (1939): The Social Function of Science, Londres, Routledge and Kegan.

Bush, Vannevar (1945): Science, the Endless Frontier, Washington D.C., U.S. Government Printing Office.

Braun, D. (1998): "The role of funding agencies in the cognitive development of science", Research Policy, vol. 27, n. 8 8, 807-821.

Brickman, Ronald (1981): "The Comparative Political Analysis of Science and Technology", Comparative Politics, vol. 13, n. ${ }^{\circ}$, pp. 479-496.

Caracostas, Paraskevas y Muldur, Ugur (1998): Society, the endless frontier. A European vision of research and innovation for the $21^{\text {ts }}$ Century, Luxemburgo, Comisión Europea, Dirección General

Recibido: 12 de julio de 2008

Aceptado: 15 de noviembre de 2008 vernment", The American Economic Review, vol. 87, n.o 2, pp. 169-172.

Cooke, Ph.; Gómez Uranga, M. y Etxebarría, G. (1997): "Regional innovation systems: Institutional and organisational dimensions", Research Policy, vol. 26, n. ${ }^{\circ} 4-5$, pp. 475-491.

Dickson, David (1984): The new politics of science, Chicago, The University of Chicago Press.

Dorado, R.; Rojo, J. M.; Triana, E. y Martínez, M. (1991): Ciencia, tecnología e industria en España, Madrid, Fundesco.

Drori, Gili S.; Meyer, John W.; Ramírez, Francisco 0. y Schofer, Evan (2003): Science in the Modern World Polity: Institutionalization and Globalization, Stanford, Standford University Press.

Elzinga, Aant y Jamison, Andrew (1995): "Changing Policy Agendas in Science and Technology", en S. Jasanoff, G. E. Markle, J. C. Petersen, y T. Pinch (eds.) (1995), Handbook of Science and Technology Studies, Thousand OaksLondres, Sage.

Fernández Carro, Remo (2002): Regímenes politicos y actividad científica: Las politicas de la ciencia en las dictaduras y las democracias, Madrid, Instituto Juan March.

Freeman, Christopher (1987): Technology policy and economic performance: Lessons from Japan, Londres, Pinter.

Godin, Benoît (2007): "National Innovation System: The System Approach in Historical Perspective", Project on the History and Sociology of STI Statistics. Working Paper no. 36, Montreal, Canadá.

González de la Fe, Teresa; Pérez Yruela, Manuel y Fernández Esquinas, Manuel (1996): "La formación de investigadores en el Plan Nacional de I+D: una aproximación evaluativo", Revista de Gestión y Análisis de Politicas Públicas, n.s 5-6. 
- (1997): "La formación y la investigación científica desde el punto de vista de los becarios de investigación", Arbor, n. ${ }^{\circ} 613$.

González de la Fe, Teresa; Torres, Cristóbal y Fernández Esquinas, Manuel (2007): "Sociología del conocimiento, de la ciencia y de la tecnología", en Pérez Yruela, Manuel (2007), La Sociología en España, Madrid, Centro de Investigaciones Sociológicas, pp. 541-563.

Gummett, Philip (1992): "Science and Technology Policy", en Hawkesworth, Mary y Maurice Kogan (eds.), Encyclopedia of Government and Politics, vol. 2, Londres-Nueva York, Rouledge, pp. 759-776.

Guston, D. H. (1996): "Principal-agent theory and the structure of science policy", Science and Public Policy, vol. 23, n. ${ }^{\circ}$, 229-240.

Guzzetti, Luca (1995): A brief history of European Union Research Policy, Bruselas, Comisión Europea.

Kleinman, Daniel Lee (1995): Politics on the endless frontier. Postwar research policy in the United States, DurhamLondres, Duke University Press.

Kraemer, Sylvia (2006): Science and Technology Policy in United States: Open Systems in Action, Nueva Jersey, Rutgers University Press.

Lafuente, Alberto y Oro, Luis (1992): El sistema español de ciencia y tecnología en el marco internacional. Evolución y perspectivas, Madrid, Fundesco.

Larèdo, Philippe y Mustar, Philippe (eds.) (2001): Research and Innovation Policies in the New Global Economy. An International Comparative Analysis, Cheltenham (Reino Unido), Edward Elgar.

López Piñero, José Maria (ed.) (1991): España. Ciencia, Madrid, Espasa Calpe.

Lundvall, Bengt-Åke (Ed.) (1992): National Systems of Innovation: Towards a
Theory of Innovation and Interactive Learning, Londres, Pinter.

Mansfield, Edwin (1966): "The Economics of Science Policy. National Science Policy: Issues and Problems", The American Economic Review, vol. 56, n. ${ }^{\text {s }}$ 1/2, pp. 476-488.

Mayor Zaragoza, Federico (ed.) (1982): Investigación científica y metas sociales. Hacia un nuevo modelo de desarrollo, Madrid, Alhambra.

- (1996): "50 años de la UNESCO", en Veintiuno: revista de pensamiento $y$ cultura, n. ${ }^{2} 29$, pp. 51-54.

Meny, Ives y Thoening, Jean-Claude (1992): Las políticas públicas, 1. a edición, Barcelona, Ariel Ciencia Política.

Moso, Mónica (1999): Origen y evolución de las políticas científicas y tecnológicas en la Comunidad Autónoma del País Vasco (1980-1998). Tesis doctoral, Departamento de Ciencia Política y de la Administración, Universidad del País Vasco.

Muñoz, Emilio y Ornia, Florencio (1986): Ciencia y Tecnología: Una oportunidad para España, Madrid, Aguilar.

Nelson, Richard (ed.) (1993): National Innovation Systems: A Comparative Analysis, Oxford, Oxford University Press.

Nieto, Alejandro (ed.) (1982): "Apuntes para una política científica: Dos años de investigación en el CSIC: 19801982", Madrid, Consejo Superior de Investigaciones Científicas.

OECD (1961): Science and the Politics of Government. The Implications of Science and Technology for National and International Affairs, Paris, OECD Publications.

- (1964): Frascati Manual. Proposed Standard Practice for Surveys of Research and Experimental Development, Paris, OECD Publications.

- (1971): Science, Growth and Society, París, OECD Publications.
- (1981): Science and Technology Policy for the 1980s, Paris, OECD Publications.

- (1994): Using patent data as science and technology indicators. Patent manual 1994, París, OECD Publications.

- (1995): Manual on the measurement of human resources devoted to S\&T. "Canberra Manual", París, OECD Publications.

- (1997): National Innovation Systems, París, OECD Publications.

- (1999): Managing National Innovation Systems, Paris, OECD Publications.

OCDE y EUROSTAT (2005): Manual de Oslo. Guía para la recogida e interpretación de datos sobre innovación, 3. edición, Madrid, Grupo Tragsa.

Olazaran, M. y Gómez Uranga, M. (eds.) (2001): Sistemas Regionales de Innovación, Zarautz (Guipúzcoa), Servicio Editorial Universidad del País Vasco.

Polanyi, Michael (1962): "The Republic of Science. Its Political and Economic Theory", Minerva, n. ${ }^{\circ}$ 1, pp. 1-20.

PREST, CSI-Ecole des Mines, CSIC-SPRITTE y SISTER (2003): A comparative study of public, semi-public and recentlyprivatised research centres in Europe, Informe final del proyecto Eurolabs. http://www.mbs.ac.uk/research/centres/engineering-policy/publications/ reports.htm.

Price, Don K. (1954): Government and Science, Nueva York, New York University Press.

Rico-Castro, Pilar (2007): La política tecnológica y sus efectos sobre el cambio de las organizaciones de I+D: El caso de los centros tecnológicos del País Vasco (1980-1999). Tesis Doctoral, Departamento de Ciencia Política y de la Administración II, Universidad Complutense de Madrid.

Rip, Arie (1994): "The Republic of Science in the 1990s", en Higher Education, vol. 28, n. 1 , pp. 3-23. 
Ronayne, Jarlath (1984): Science in Government, Victoria (Australia), Edward Arnold.

Ruivo, Beatriz (1994): "Phases and paradigms of science policy?", Science and Public Policy, vol. 21, n. 3 , pp. 157-163.

Quintanilla, Miguel Ángel (ed.) (1992): "El Sistema Español de Ciencia y Tecnología (Proyecto EPOC)", Arbor, n. ${ }^{\text {os }}$ 444-445.

Salomon, Jean-Jacques (1970): Science and Politics, Cambridge (Ma), The MIT Press.

Sanz-Menéndez, Luis (1997): Estado, Ciencia y Tecnología en España: 19391997, Madrid, Alianza Universidad.
Sanz-Menéndez, Luis y Cruz-Castro, Laura (2005): "Explaining the Science and Technology Policies of Regional Governments", Regional Studies, vol. 39, n. ${ }^{\circ}$, pp. 939-954.

Sanz-Menéndez, Luis y Santesmases, M. ${ }^{a}$ Jesús (1996): "Ciencia y política: Interacciones entre el Estado y el sistema de investigación", Zona Abierta, 75/76, pp. 1-20.

Sebastián, Jesús (1985): "El Sistema de Prioridades en Política Científica", Arbor, n. ${ }^{\circ} 475$.

Shils, Edward (ed.) (1968): Criteria for Scientific Development: Public Policy and National Goals, Cambridge (Ma)Londres, MIT Press.
Solingen, Etel (1993): "Between the Markets and the State. Scientists in Comparative Perspective", Comparative Politics, vol. 26, n. ${ }^{\circ} 1, \mathrm{pp}$. 31-51.

Tisdell, C. A. (1981): Science and Technology Policy. Priorities of Governments, Londres, Chapman and Hall.

Van der Meulen, Barend (1998): "Science Policies as principal-agent games; Institutionalization and path dependency in the relation between government and science", Research Policy, n. ${ }^{\circ} 27, \mathrm{pp} .397-414$.

Ziman, John (1984): An Introduction to Science Studies, Cambridge University Press. 